\title{
Plurality inferences are scalar implicatures: Evidence from acquisition *
}

\author{
Lyn Tieu \\ École Normale Supérieure \\ Jacopo Romoli \\ University of Ulster
}

\author{
Cory Bill \\ Macquarie University \\ Stephen Crain \\ Macquarie University
}

\begin{abstract}
This paper provides novel experimental evidence for a scalar implicature approach to the plurality inferences that are associated with English plural morphology (Emily fed giraffes $\rightsquigarrow$ Emily fed more than one giraffe). Using a Truth Value Judgment Task, we show that both adults and 4-5-year-old children compute more plurality inferences in upward-entailing than downward-entailing environments, but children compute fewer plurality inferences overall than adults do. These findings are consistent with previous research demonstrating children's relative insensitivity to scalar implicatures. We discuss the implications of these findings for theories of plurality inferences, and for the acquisition of scalar inferences more generally.
\end{abstract}

Keywords: plurality, plurality inferences, scalar implicatures, language acquisition, bare plurals

\section{Introduction}

\subsection{Plurality inferences as scalar implicatures}

The plural-singular distinction is the source of a long-standing puzzle (see Sauerland 2003; Sauerland, Andersen \& Yatsushiro 2005; Spector 2007; Zweig 2009 and Magri 2014 for discussion). In brief, the puzzle is as follows: a plural sentence like (1-a) appears equivalent to (1-b) and different from its singular counterpart (1-c),

* For helpful feedback and discussion, we would like to thank Emmanuel Chemla, Giorgio Magri, Uli Sauerland, Florian Schwarz, Benjamin Spector, and the audiences at SALT24 at NYU and the SNEWS workshop at MIT. We are also grateful to the families who participated in the study at the $\mathrm{CCD}$ at Macquarie University. The research leading to these results was supported by the Australian Research Council Centre of Excellence in Cognition and its Disorders (CE110001021), the European Research Council under the European Union's Seventh Framework Programme (FP/2007-2013) / ERC Grant Agreement n.313610, and by ANR-10-IDEX-0001-02 PSL* and ANR-10-LABX-0087 IEC. 
Plurality inferences are scalar implicatures

suggesting that English plural morphology is associated with a meaning like 'more than one' (Lasersohn 1995, among others).
a. Emily fed giraffes.
b. Emily fed more than one giraffe.
c. Emily fed a giraffe.

If (1-a) just meant (1-b), however, we would expect that embedding (1-a) under negation as in (2-a) would result in a corresponding "not more than one" reading (2-b). However, example (2-a) is not generally interpreted as having the same meaning as (2-b), but, rather, is better paraphrased as the negation of the singular $(1-c)$, as in (2-c).
a. Emily didn't feed giraffes.
b. Emily didn't feed more than one giraffe.
c. Emily didn't feed a (single) giraffe.

The shape of the puzzle is therefore the following: the interpretation of a positive sentence with plural morphology like (1-a) appears to give rise to a more-thanone inference in positive contexts, but this inference tends to disappear if (1-a) is embedded under negation. We will refer to (1-b) as the 'plurality inference' of the sentence (1-a).

A successful response to this puzzle treats plurality inferences as a type of scalar implicature (Sauerland 2003; Sauerland et al. 2005; Spector 2007; Zweig 2009). For instance, Spector (2007) argues that plurality inferences arise as the consequence of a higher-order type of scalar implicature that involves a comparison of the plural and the singular, where the singular has already been enriched with its own scalar implicatures (see also Magri 2014). ${ }^{1}$ More specifically, Spector's idea is the following: both a sentence like (1-a) and its singular counterpart (1-c) have a weak literal meaning that can be paraphrased as in (3-a). On the one hand, the singular (1-c) is typically compared to a sentence like (3-b), yielding the scalar implicature in (4-a). The plural (1-a), on the other hand, is compared to the singular that has been enriched with its scalar implicature, namely (4-a). As Spector shows, when (4-a) is negated, the plurality inference is generated, yielding (4-b). ${ }^{2}$

1 Magri (2014) extends Spector's account of bare plurals to plural definites and conjunction. We restrict our attention to bare plurals in this study.

2 More formally, the semantics of the singular and plural can be represented as in (i-a) (consider a model with three giraffes, Jill, Mary, and Fran). The singular is then compared to (i-b), yielding the standard scalar implicature in (ii-a). By contrast, the plural is compared to the singular enriched with its scalar implicature, namely (ii-a). The negation of (ii-a) gives rise to the plurality inference in (ii-b). 
a. Emily fed one or more giraffes.

b. Emily fed more than one giraffe.

a. Emily fed one but not more than one giraffe.

b. Emily fed more than one giraffe.

Given that scalar implicatures are typically not derived when scalar terms appear in downward-entailing contexts, the scalar implicature approach predicts the pattern of interpretation observed in (1) and (2). Moreover, the scalar implicature approach can account for an additional reading of (2-a) that excludes singularity, namely that in (5) (which is typically read with emphasis on the plural $-s$ ); this involves postulating a local scalar implicature under the scope of negation. Such a reading is correctly predicted to be marked.

Emily didn't feed giraffes, because she fed only one!

\subsection{Predictions for acquisition}

If plurality inferences are derived as a kind of scalar implicature, as we have described, the pattern of children's plurality inferences is expected to mirror their performance with other scalar implicatures. Despite considerable variation in the reported rates of children's success with scalar implicatures, a consistent finding is that children compute scalar implicatures less than adults do (Gualmini, Crain, Meroni, Chierchia \& Guasti 2001; Chierchia, Crain, Guasti \& Thornton 2001; Noveck 2001; Papafragou \& Musolino 2003, among others). For example, children typically accept sentences such as (6-a) in situations where the stronger alternative (6-b) is also true. This observation has usually been taken to indicate that children fail to compute the implicature in (6-c).
a. Emily fed some of the giraffes.
b. Emily fed all of the giraffes.
c. Emily fed some but not all of the giraffes.

If plurality inferences are scalar implicatures, therefore, we would expect children to also fail to compute (1-b) from (1-a). To assess this possibility, Sauerland et al.

(i) $\quad$ a. $\quad[$ giraffes $]]=\llbracket[$ giraffe $]=\{j, m, f, j \oplus m, j \oplus f, f \oplus m, j \oplus m \oplus f\}$

b. $\quad[$ more than one giraffe] $]=\{j \oplus m, j \oplus f, f \oplus m, j \oplus m \oplus f\}$

(ii) $\quad$ a. $\quad$ [giraffe $] \wedge \neg[$ more than one giraffe $]]=\{j, m, f\}$

b. $\quad[$ giraffes $] \wedge \neg([$ giraffe $] \wedge \neg \neg[$ more than one giraffe $])=$ $\{j \oplus m, j \oplus f, f \oplus m, j \oplus m \oplus f\}$ 
Plurality inferences are scalar implicatures

(2005) tested 3-5-year-olds' computation of plurality inferences in polar questions such as (7).

(7) Does a dog have tails?

The authors found that children accepted these more often than adults did. Given the parallel in children's performance on scalar implicatures and plurality inferences, this finding would appear to provide evidence for the scalar implicature approach. As the authors point out however, the study had some potential limitations (see also Pearson, Khan \& Snedeker (2011) for similar criticisms). First, plurality inferences typically disappear in polar questions. Second, the experimental stimuli involved generic interpretations, which could have been misinterpreted by children as containing dependent plurals. For example, children might have interpreted (7) as Do dogs have tails? The study by Sauerland et al. (2005) therefore goes in the direction of supporting the scalar implicature approach, but several potential confounds of the study ultimately render it inconclusive. In the next section, we present an experiment that avoids the potential problems of the Sauerland et al. (2005) study.

\section{Experiment}

We designed a Truth Value Judgment Task (Crain \& Thornton 1998) to assess the interpretation that adults and children assign to declarative sentences with singulars and plurals, in both upward and downward-entailing environments. Comparing children and adults on the interpretation of these sentences allowed us to test the scalar implicature approach along the lines of Sauerland et al. 2005. Testing the interpretation of declarative sentences allowed us to avoid the potential problem that was associated with the disappearance of plurality inferences in polar questions in the Sauerland et al. 2005 study. Examining both upward- and downward-entailing environments enabled us to further test the scalar implicature approach advanced by Spector (2007), which predicts the disappearance of plurality inferences in downward-entailing environments.

\subsection{Methods}

\subsubsection{Participants}

The participants were 30 English-speaking children and 44 adult native speakers of English. Only those participants who passed at least 6 of the 8 control trials were included in the analysis. We report here the data from the 28 children $(4 ; 01-5 ; 09$, $\mathrm{M}=4 ; 11)$ and the 43 adults who passed the control trials. 
Tieu, Bill, Romoli, Crain

\subsubsection{Procedure}

Participants watched a series of short stories told by an experimenter, through pictures on a laptop computer. The pictures were presented in the form of a Powerpoint presentation. Participants were introduced to a puppet who they were told would interact with them via webcam. In fact, videos of the puppet's lines were prerecorded, and were played at various points during the stories to create the ruse that the puppet was participating live via webcam. Following each story, the puppet appeared on the screen and was asked a question about the story. The participant's task was to judge the puppet's answers to the questions. Child participants were given a scoresheet with two columns, one headed by a "happy face" and one headed by a "sad face"; the children were asked to place a stamp under the "happy face" if the puppet had answered the question correctly, and to place a stamp under the "sad face" if the puppet's answer was wrong. Adult participants had similar scoresheets but were asked to place a checkmark in the appropriate column; adults also had a third column in which to write short justifications for their responses. The task generally took about 20 minutes for children and between 10-15 minutes for adults.

\subsubsection{Materials}

We adopted a 2x2x2 design with three factors: group (adults vs. children), number (singular vs. plural-between subjects), and monotonicity (upward- vs. downwardentailing-within subjects). Participants were randomly assigned to either the singular or plural condition. In total, there were six test stories and eight control stories, which were presented in pseudo-randomized order. Three of the critical test stories were associated with a positive (plural or singular) sentence, and three with a negative (plural or singular) sentence.

Consider first the upward-entailing condition. On these three trials, the main character in the story executed an action on only one object from a set of objects; in the plural condition, participants heard a test sentence with a bare plural, while participants heard a singular noun phrase in the singular condition. See (8) for an example test item and see Figure 1 for the accompanying final image.

(8) Story: Emily is visiting the pig farm today. It's lunchtime for the pigs. Emily has an apple, and that's just enough to feed the first pig! Oh no! What about the other pigs? The farmer says, "That's okay, Emily! I'll feed the others later!" So in the end, Emily only fed this pig!

EXPERIMENTER: Hey Ellie, what happened in the story?

a. PUPPET: Emily fed pigs! (target: NO)

Plural condition

b. PUPPET: Emily fed a pig! (target: YES)

Singular condition 


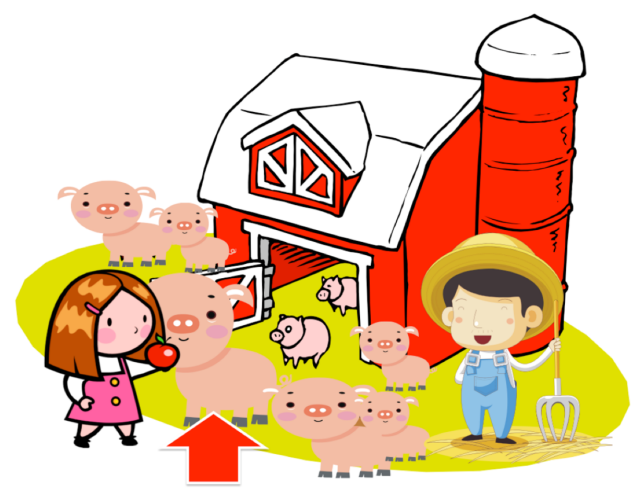

Figure 1 Final image accompanying the plural test sentence Emily fed pigs and the singular test sentence Emily fed a pig.

As seen in (8) and in Figure 1, emphasis was placed on the single pig that was fed; to make it very clear that only that particular pig was fed, the prompt contained the focus particle only and a definite determiner (i.e. Emily only fed this pig!); moreover, a red arrow was added to the picture and the experimenter gestured to the pig when referring to it. $^{3}$

In the plural condition, participants who computed the plurality inference in the upward-entailing condition were expected to reject the sentence Emily fed pigs, since it was false that Emily fed more than one pig. The singular test sentence was true in the context, however. Thus we expected different responses to singular and plural sentences in the upward-entailing condition.

The stories for the downward-entailing condition were the same in structure, but the test sentences included negation. Here we expected participants to reject the test sentences in both the singular and plural conditions. In other words, the difference between singular and plural should be neutralized under negation. We did, however, expect some proportion of participants to accept the negative test sentences in such contexts if they were able to access a meaning like (5), Emily didn't feed giraffes, because she fed only one! See (9) for an example test item and Figure 2 for the accompanying final image.

(9) Story: Emily is visiting the zoo today. It's lunchtime for the animals. Emily has just enough food to feed this very tall giraffe! Oh no! What about the other giraffes? The zookeeper says, "That's okay, Emily! I'll feed the others

3 This was also meant to highlight the episodic nature of the description, i.e. that there was a single event involving the feeding of one pig in particular, and to avoid a possible activity reading of the plural test sentence, e.g., Emily was pig-feeding. 
Tieu, Bill, Romoli, Crain

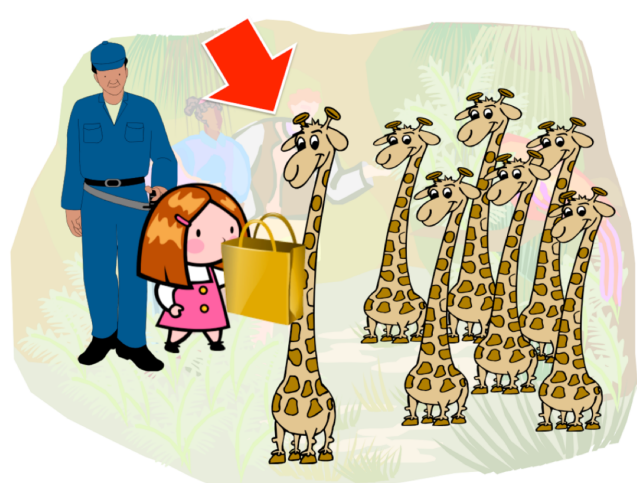

Figure 2 Final image accompanying either plural Emily didn't feed giraffes or singular Emily didn't feed a giraffe

later!" So in the end, Emily only fed this giraffe!

EXPERIMENTER: Hey Ellie, what happened in the story?

a. PUPPET: Emily didn't feed giraffes! (target: NO) Plural condition

b. PUPPET: Emily didn't feed a giraffe! (target: NO) Singular condition ${ }^{4}$

Both the plural and the singular test conditions also included two positive and two negative control items. These control items were designed to elicit the opposite responses to those of the critical test trials. In addition to helping to make sure participants stayed on task, the addition of these controls allowed us to ensure that the participants could give both yes-and no-responses to the test sentences, in the appropriate situations.

In the plural condition, participants heard two positive plural sentences in contexts that satisfied the plurality inference (e.g., (10)), and they heard two negative plural sentences in contexts that did not satisfy the plurality inference (e.g., (11)):

Plural-positive control:

Context: Sammy painted two birds.

Test sentence: Sammy painted birds. (target: YES)

4 Notice that this sentence potentially has an interpretation on which the indefinite takes scope over negation: there is a giraffe that Emily didn't feed. In this case, the sentence would be true in the given context. To avoid this specific/wide-scope reading as much as possible, the relevant pictures, for example that in Figure 2, contained multiple identical animals that were not fed; this way, none of them would stand out from the others. As we will see below, adults and children generally did not interpret the sentence in this way. 
Plurality inferences are scalar implicatures

(11) Plural-negative control:

Context: Sammy drew one cat.

Test sentence: Sammy didn't draw dogs. (target: YES)

In the singular condition, two control stories made the positive singular test sentences false (e.g., (12)), and two control stories made the negative test sentences true (e.g., (13)).

(12) Singular-positive control:

Context: Sammy painted one bird.

Test sentence: Sammy painted a tree. (target: NO)

(13) Singular-negative control:

Context: Sammy drew one cat.

Test sentence: Sammy didn't draw a dog. (target: YES)

All participants also received four control sentences that included negation but contained a definite noun phrase rather than a bare plural or an indefinite $a$-noun phrase. These trials ensured that child participants had no difficulty interpreting negation independently of the plural or $a$-NP, and they allowed us to ensure that the participants remained on task. The negation control trials could be associated with either a yes- or no-target; the targets were selected based on participants' responses on the test trials. These enabled us to balance the overall number of yesand no-responses. An example of the negation controls is provided in (14).

(14) Negation control:

Context: Sally had a choice between the chocolate and the apple, and in the end ate the apple.

a. Yes-target: Sally didn't eat the chocolate.

b. No-target: Sally didn't eat the apple.

To summarize, each participant received 6 critical test items: 3 corresponding to plural or singular sentences in upward-entailing environments, and 3 corresponding to plural or singular sentences in downward-entailing environments. In addition, each participant received 8 control items: 2 positive and 2 negative sentences that elicited the opposite truth value from the corresponding critical trials, and 4 negation controls. The 14 items were presented in pseudo-randomized and counterbalanced order. 


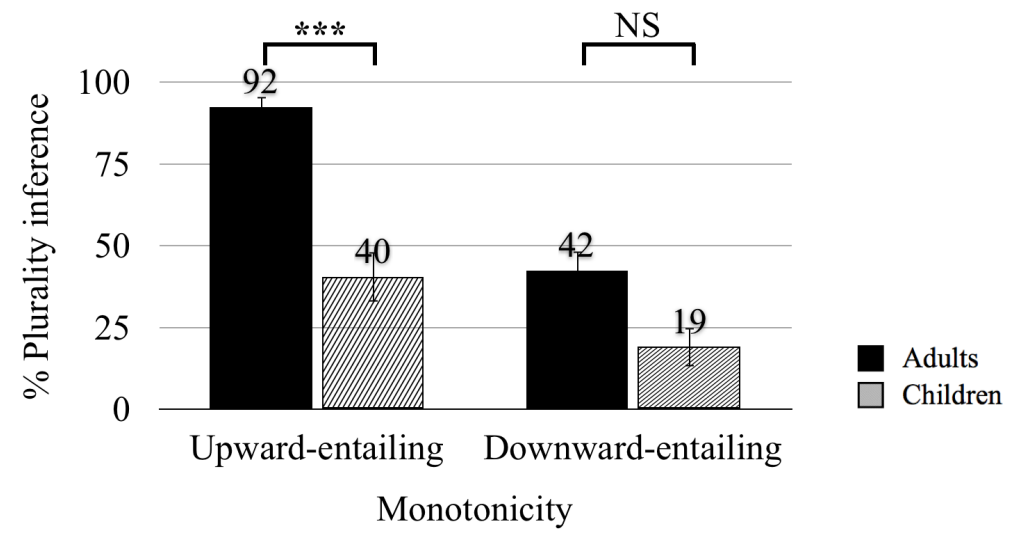

Figure 3 Rates at which participants computed the plurality inference in the plural condition (computation of plurality inferences corresponded to no-responses in the upward-entailing condition and to yes-responses in the downward-entailing condition).

\subsection{Results}

A 2x2x2 ANOVA on participants' responses revealed a significant main effect of monotonicity $(F(1,134)=114.81, p<.001)$, a significant main effect of number $(F(1,134)=21.52, p<.001)$, no main effect of $\operatorname{group}(F(1,134)=3.15, p=.08)$, and a significant interaction between monotonicity, number, and group $(F(1,134)=$ $19.55, p<.001)$.

\subsubsection{Plural conditions}

Consider first the plural conditions. Figure 3 displays the rates at which participants computed the plurality inference; note that computation of plurality inferences corresponded to no-responses in the upward-entailing (positive) condition and to yes-responses in the downward-entailing (negative) condition.

The observation of fewer plurality inferences from children than adults in the upward-entailing condition is consistent with previous findings that children compute scalar implicatures less than adults do. In the downward-entailing condition, children did not differ significantly from adults, with some proportion of both adults and children appearing to access the interpretation in (5), made available by locally computing the plurality inference in the scope of negation.

The follow-up justifications produced by participants were used to confirm that they were indeed accessing one of the possible target interpretations. Some examples of justifications from the child participants are provided in (15) through (18). 
Plurality inferences are scalar implicatures

(15) Justifications for yes-responses in upward-entailing condition (60\% of responses)

(Test sentence: Emily fed pigs.)

a. "Because she feed a pig."

b. "Because Emily fed pigs."

c. "Because she said the pig has been feeded, and that happened."

(16) Justifications for no-responses in upward-entailing condition (40\% of responses)

(Test sentence: Emily fed pigs.)

a. "Because she didn't feed all of them."

b. "Because she didn't feed pigs, she only fed a pig."

c. "Because she was only going to feed that big fat pig."

(17) Justifications for no-responses in downward-entailing condition (81\% of responses)

(Test sentence: Emily didn't feed giraffes.)

a. "Because she said Emily didn't feed the giraffes, and she did."

(18) Justifications for yes-responses in downward-entailing condition (19\% of responses)

(Test sentence: Emily didn't color diamonds.)

a. "Because she only did one diamond."

b. "Because she colored that one, but not the other ones."

\subsubsection{Singular conditions}

Figure 4 shows the rate of yes-responses on both upward- and downward-entailing singular conditions. Both children and adults generally accepted in the positive condition but rejected in the negative condition. Children were adult-like in both the upward-entailing and downward-entailing conditions.

Examples of children's justifications for the singular condition are provided in (19) and (20).

(19) Justifications for yes-responses in upward-entailing condition (100\% of responses)

(Test sentence: Emily fed a pig.)

a. "Because she gave the apple to one pig."

b. "Because Emily fed the pig." 


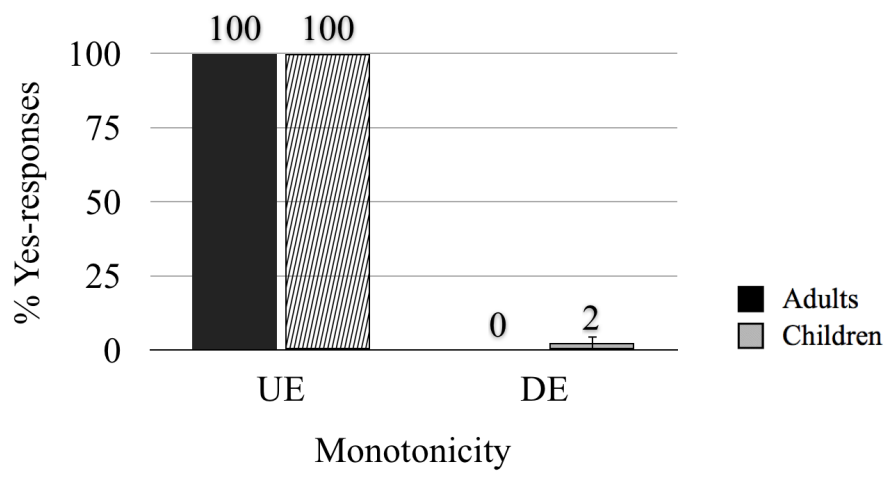

Figure 4 Rate of acceptance in singular conditions.

(20) Justifications for no-responses in downward-entailing condition (98\% of responses)

(Test sentence: Emily didn't feed a giraffe.)

a. "Because she did feed a giraffe."

\section{Discussion}

The experimental results can be summed up by two main observations. The first main finding is that children and adults compute plurality inferences more in upwardentailing than in downward-entailing linguistic environments. On the scalar implicature approach, plurality inferences should exhibit precisely this hallmark of standard scalar implicatures: they should arise in upward-entailing environments, and disappear in downward-entailing ones. This is indeed what we found.

The second and more important finding lies in the comparison of children's and adults' performance on the task. We found that children computed plurality inferences less often than adults did. Eight out of the 14 children in the plural condition accepted the test sentences on at least two of the three critical upwardentailing trials, apparently failing to access the "more than one" meaning of the plural. This finding is consistent with previous findings from the developmental literature on children's computation of scalar implicatures, and provides further support for the scalar implicature approach to plurality inferences. If plurality inferences and scalar implicatures are one and the same, then it is not surprising that children exhibit the same developmental pattern across the two phenomena. Moreover, the present study overcame some possible limitations of the previous study by Sauerland (2005): first, we examined upward-entailing contexts, which allowed us to find a clear difference between children and adults' performance, and 
second, there is no clear way in which our stimuli could have been misinterpreted as involving dependent plurals.

Notice that six of the 14 children in the plural condition rejected the test sentences on at least two of the three critical trials in the upward-entailing condition. These children appear to have successfully computed plurality inferences. A future study might involve a within-subject design that would enable us to compare children's performance on plurality inferences with that of other kinds of scalar implicatures (e.g., involving some vs. all, may vs. must, numerals, etc.). What is important for our present purposes is that children calculated plurality inferences less than adults did. It is worth questioning, however, whether plurality inferences might differ from other scalar implicatures in more fine-grained ways, as it appears that children do not treat all scalar inferences alike. It has been observed for example that children perform better on scalar implicatures associated with numerals than on the not-all implicature associated with some (Papafragou \& Musolino 2003). Might we expect children's performance on plurals to pattern more with their performance on numerals or more with their performance on scalar quantifiers such as some?

While we must leave the assessment of children's responses using a withinsubject design for future study, we would like to make a brief connection here between the current findings and those of the existing developmental literature on scalar implicatures. One connection worth pursuing involves the proposal that children's difficulty with scalar implicatures lies in a difficulty in accessing alternatives (see Gualmini et al. 2001; Reinhart 2004; Barner, Brooks \& Bale 2011). It has been observed that children are better at computing scalar implicatures when the required alternatives are made more easily accessible to them, either through explicit mention in the discourse or as substrings of the test sentences themselves (Gualmini et al. 2001; Chierchia et al. 2001; Barner et al. 2011; Zhou, Romoli \& Crain 2013). This observation has prompted the proposal that children succeed on inferences that do not involve retrieval of the relevant alternatives from the lexicon (for further discussion see Tieu, Romoli, Zhou \& Crain under review). In the case of plurality inferences, if alternatives are what are relevant, then there are at least two crucial ingredients to children's success. First, the child must learn the co-scalar status of the singular and the plural. After all, on the scalar implicature approach to plurality, it is the negation of the enriched singular that gives rise to the plurality inference. The child must therefore have knowledge of these competitors and must know how to exhaustify the alternatives. Second, the child must be able to retrieve the singular from the lexicon in order to compute the plurality inference. Difficulties with computing the plurality inference could lie in either ingredient.

There are reasons to think that learning the relevant relationship between the singular and the plural may be equally if not more difficult than learning the co-scalar status of full lexical items such as some and all. It is not so difficult to imagine 
children encountering evidence for the not-all implicature, e.g., I said you could have some of the cookies, not all of them! It is possible to contrast the two by simply replacing an instance of one with an instance of the other. It is less obvious what kind of evidence would signal the difference between singular and plural for children. The difference between the (regular) plural and the singular in English is signaled by the presence of the plural marker $-s$ vs. the combination of the absence of the plural marker and the presence of an indefinite determiner. In this sense the co-scalar status of the two is much more abstract than in the case of some vs. all; there isn't an obvious sense in which one can be contrasted with the other. ${ }^{5}$

\section{Conclusion}

This study set about testing the scalar implicature approach to plurality inferences. Our main findings were the following. First, more plurality inferences were computed in upward-entailing than in downward-entailing environments for both children and adults. Second, children computed plurality inferences less often than adults did. All in all, our findings strongly support a scalar implicature approach to plurality inferences. The observed differences in behavior between children and adults are typical of findings from previous developmental studies on scalar implicatures. We have tried to make a brief connection with existing proposals about children's difficulty with scalar implicatures and have suggested a potential future study that would explicitly compare children's performance on plurality inferences with their performance on other kinds of scalar implicatures. Whatever the source of difficulty for the child, our hope is that it will prove fruitful to regard plurality inferences as a piece of the puzzle. Identifying how children's performance on plurality inferences both mirrors and differs from other kinds of scalar inferences will eventually yield a fuller picture of the nature of different scalar inferences, and how they are acquired by child language learners.

\section{References}

Barner, David, Neon Brooks \& Alan Bale. 2011. Accessing the unsaid: the role of scalar alternatives in children's pragmatic inferences. Cognition 188. 87-96.

5 With respect to overt morphological marking of the plural, it may prove insightful to investigate the development of plurality inferences in languages that do not morphologically mark the plural on the noun; alternatively, one might investigate the development of plurality inferences in the context of irregular nouns in English. Thanks to Scott AnderBois for raising these as potential avenues for future research. 
Plurality inferences are scalar implicatures

Chierchia, Gennaro, Stephen Crain, Maria Teresa Guasti \& Rosalind Thornton. 2001. 'Some' and 'or': A study on the emergence of logical form. In S. Catherine Howell, Sarah A. Fish \& Thea Keith-Lucas (eds.), Boston University Conference on Language Development (BUCLD) 24, 22-44. Somerville, Massachusetts: Cascadilla Press.

Crain, Stephen \& Rosalind Thornton. 1998. Investigations in Universal Grammar: A Guide to Experiments on the Acquisition of Syntax and Semantics. Cambridge, Massachusetts: MIT Press.

Gualmini, Andrea, Stephen Crain, Luisa Meroni, Gennaro Chierchia \& Maria Teresa Guasti. 2001. At the semantics/pragmatics interface in child language. In Rachel Hastings, Brendan Jackson \& Zsofia Zvolenszky (eds.), Semantics and Linguistic Theory (SALT) 11, 231-247. Cornell University, Ithaca, NY: CLC Publications.

Lasersohn, Peter. 1995. Plurality, Conjunction and Events. Dordrecht, The Netherlands: Kluwer Academic Publishers.

Magri, Giorgio. 2014. An account for the homogeneity effects triggered by plural definites and conjunction based on double strengthening. In Salvatore Pistoia Reda (ed.), Pragmatics, Semantics and the Case of Scalar Implicatures, 99-145. Palgrave Macmillan.

Noveck, Ira. 2001. When children are more logical than adults: experimental investigations of scalar implicatures. Cognition 78(8). 165-188.

Papafragou, Anna \& Julien Musolino. 2003. Scalar implicatures: experiments at the semantics-pragmatics interface. Cognition 86. 253-282.

Pearson, Hazel, Manizeh Khan \& Jesse Snedeker. 2011. Even more evidence for the emptiness of plurality: An experimental investigation of plural interpretation as a species of scalar implicature. In David Lutz \& Nan Li (eds.), Semantics and Linguistic Theory (SALT) 20, Cornell University Ithaca, NY: CLC Publications. Reinhart, Tanya. 2004. The processing cost of reference set computation: Acquisition of stress shift and focus. Language Acquisition 12(2). 109-155.

Sauerland, Uli. 2003. A new semantics for number. In Robert B. Young \& Yuping Zhou (eds.), Semantics and Linguistic Theory (SALT) 13, 258-275. Cornell University Ithaca, NY: CLC Publications.

Sauerland, Uli. 2005. DP is not a scope island. Linguistic Inquiry 36(2). 303-314.

Sauerland, Uli, Jan Andersen \& Kazuko Yatsushiro. 2005. The plural is semantically unmarked. In Stephan Kepser \& Marga Reis (eds.), Linguistic Evidence: Empirical, Theoretical, and Computational Perspectives, 413-434. Mouton de Gruyter.

Spector, Benjamin. 2007. Aspects of the pragmatics of plural morphology: On higher-order implicatures. In Uli Sauerland \& Penka Stateva (eds.), Presupposition and Implicature in Compositional Semantics, 243-281. Palgrave.

Tieu, Lyn, Jacopo Romoli, Peng Zhou \& Stephen Crain. under review. Children's 
knowledge of free choice inferences .

Zhou, Peng, Jacopo Romoli \& Stephen Crain. 2013. Chidren's knowledge of alternatives. In Todd Snider (ed.), Semantics and Linguistic Theory (SALT) 23, 632-651. Cornell University Ithaca, NY: CLC Publications.

Zweig, Eytan. 2009. Number neutral bare plurals and the multiplicity agreement. Linguistics and Philosophy 32. 353-407.

Lyn Tieu

Laboratoire de Sciences Cognitives

École Normale Supérieure

29 rue d'Ulm

75005 Paris France

lyn.tieu@gmail.com

Jacopo Romoli

Room 17E10, School of Communication

University of Ulster, Jordanstown Campus

Shore Road

Newtownabbey

Co. Antrim BT37 0QB

jacopo.romoli@gmail.com
Cory Bill

Department of Cognitive Science 16 University Avenue

Macquarie University

NSW 2109 Australia

cory.bill@mq.edu.au

Stephen Crain

Department of Linguistics

Australian Hearing Hub

16 University Avenue

Macquarie University

NSW 2109 Australia

stephen.crain@mq.edu.au 